\title{
A 2.5-mm outer diameter photoacoustic endoscopic mini-probe based on a highly sensitive PMN-PT ultrasonic transducer
}

Joon-Mo Yang, Ruimin Chen, Christopher Favazza, Junjie Yao, Qifa Zhou, et al.

Joon-Mo Yang, Ruimin Chen, Christopher Favazza, Junjie Yao, Qifa Zhou, K. Kirk Shung, Lihong V. Wang, "A 2.5-mm outer diameter photoacoustic endoscopic mini-probe based on a highly sensitive PMN-PT ultrasonic transducer," Proc. SPIE 8223, Photons Plus Ultrasound: Imaging and Sensing 2012, 82233M (23 February 2012); doi: 10.1117/12.909207

SPIE. Event: SPIE BiOS, 2012, San Francisco, California, United States 


\title{
A 2.5-mm outer diameter photoacoustic endoscopic mini-probe based on a highly sensitive PMN-PT ultrasonic transducer
}

\author{
Joon-Mo Yang ${ }^{1}$, Ruimin Chen ${ }^{2}$, Christopher Favazza ${ }^{1}$, Junjie Yao ${ }^{1}$, Qifa Zhou ${ }^{2}$, K. Kirk Shung ${ }^{2}$, \\ and Lihong V. Wang ${ }^{1 *}$ \\ ${ }^{1}$ Optical Imaging Laboratory, Department of Biomedical Engineering, Washington University in St. \\ Louis, One Brookings Drive, Campus Box 1097, St. Louis, MO 63130, USA \\ ${ }^{2}$ Ultrasonic Transducer Resource Center, Department of Biomedical Engineering, University of \\ Southern California, 1042 Downey Way, University Park, DRB 130, Los Angeles, CA 90089, USA
}

\begin{abstract}
We have developed a $2.5-\mathrm{mm}$ outer diameter photoacoustic endoscopic mini-probe to use in the instrument channel (typically 2.8 or $3.7 \mathrm{~mm}$ in diameter) of standard video endoscopes. To achieve adequate signal sensitivity, we fabricated a focused ultrasonic transducer using a highly-sensitive PMN-PT piezoelectric material. We quantified the PMN-PT transducer's operating parameters and validated the mini-probe's endoscopic imaging capability through an $e x$ vivo imaging experiment on a rat colon.
\end{abstract}

Keywords: Photoacoustic endoscopy, mini-probe, photoacoustic tomography, PMN-PT, ultrasonic transducer, gastrointestinal tract, colonoscopy.

\section{INTRODUCTION}

Photoacoustic endoscopy (PAE) ${ }^{1-4}$ could be a useful clinical tool because it possesses many of the strengths of photoacoustic tomography (PAT) ${ }^{1,5-10}$. PAT is a novel tomographic imaging technique that reveals optical absorptionbased image contrast by detecting acoustic waves induced by rapid optical absorption of target tissue, called the photoacoustic (PA) effect. PAT provides functional physiological information ${ }^{5-15}$ and high-resolution at super depths (the optical diffusive regime) far exceeding the penetration limits of conventional optical imaging. To date, a penetration depth more than $\sim 1 \mathrm{~cm}$ has been demonstrated in human subjects ${ }^{16-18}$. Angiographic or lymphangiographic imaging capabilities are clinically relevant because such images can provide vital information for disease staging ${ }^{18-21}$. PAT can provide images with endogenous contrast or can be enhanced by numerous contrast agents ${ }^{6,10}$. Hence, embodying PAT in an endoscopic device could have great impact because many important diseases that arise in the gastrointestinal tract can be treated with endoscopic procedures. As endoscopic ultrasound (EUS) mini-probe currently plays an important clinical role ${ }^{22,23}$, development of a similar mini-probe based on PAT $^{1,2,9,10,24}$ is an important next step.

Typically, instrument (or accessory) channels of clinical video endoscopes have diameters of 2.8 or $3.7 \mathrm{~mm}$ and a channel length of $\sim 1-2 \mathrm{~m}$. To use in the 2.8-mm diameter channel, for example, the mini-probe's outer diameter should be less than $2.5 \mathrm{~mm}$ as are currently used clinical EUS mini-probes ${ }^{22,23}$. In this study, we implemented a 2.5 -mm outer diameter PAE mini-probe with a flexible section (or insertion tube) length of $\sim 2 \mathrm{~m}$. Importantly, the 2.5 -mm diameter includes the entire outer sheath and, to our knowledge, is the smallest outer diameter among reported PAE probes that have been fully catheterized. The new endoscopic probe also works on the same rotating mirror-based mechanical scanning mechanism as described in our previous reports ${ }^{2-4}$; however, the length of the rigid distal section has been reduced to $\sim 35 \mathrm{~mm}$. Also, to achieve better signal sensitivity, we utilized a highly-sensitive PMN-PT piezoelectricmaterial for the ultrasonic (US) transducer. The implemented US transducer has a $\sim 1.8-\mathrm{mm}$ aperture and $\sim 33-\mathrm{MHz}$

*Corresponding author: lhwang@biomed.wustl.edu

Photons Plus Ultrasound: Imaging and Sensing 2012, edited by Alexander A. Oraevsky, Lihong V. Wang, Proc. of SPIE Vol. 8223, 82233M - (C) 2012 SPIE · CCC code: 1605-7422/12/\$18 - doi: 10.1117/12.909207 
center frequency $(\sim 60 \%$ fractional bandwidth). The presented experimental results quantify the PMN-PT transducer's operating parameters and demonstrate the PA mini-probe's endoscopic imaging capability.

\section{MATERIALS AND METHODS}

\subsection{5-mm outer diameter PA mini-probe}

The implemented mini-probe has a similar structure to our previous probe ${ }^{2-4}$, which adopts a coaxially-aligned optical illumination and US detection scheme and employs a micromotor-based mechanical scanning mechanism [Fig. 1(a)]. The main feature of this probe is that a dielectric-coated fused silica mirror (BB05-E02, Thorlabs) with a $45^{\circ}$ deflected reflection surface is employed as the principle component of the scanning mechanism (called a scanning mirror). The mirror reflects both the laser beam and acoustic waves, and performs rotational scanning at a B-scan frame of $\sim 4 \mathrm{~Hz}$, driven by the torque from the micromotor (gear ratio, 254:1; Namiki Precision). The optical fiber and US transducer unit [Fig. 1(b)] and the mechanical unit [Fig. 1(c)] are the two main constituents of the probe, and they are connected by a $\sim 70 \mu \mathrm{m}$ thick, optically and acoustically-transparent plastic (PET) tube which forms an imaging window [Fig. 1(d)]. The micromotor's four electric wires pass through the imaging window, which partially blocks the angular fieldof-view by $50^{\circ}$. The endoscope's proximal section (i.e., flexible section), which includes the optical fiber and electric wires, is encapsulated in a $\sim 2 \mathrm{~m}$ long flexible plastic tube [Fig. 1(e)]. More information on the endoscope's imaging principle is available in our previous reports ${ }^{2-4}$.

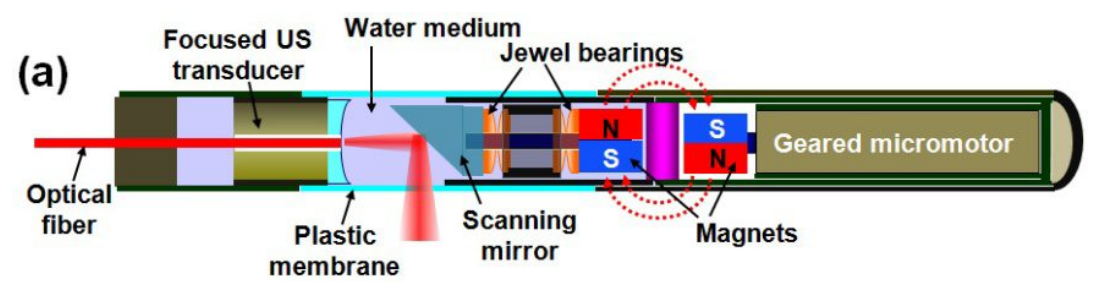

(b)
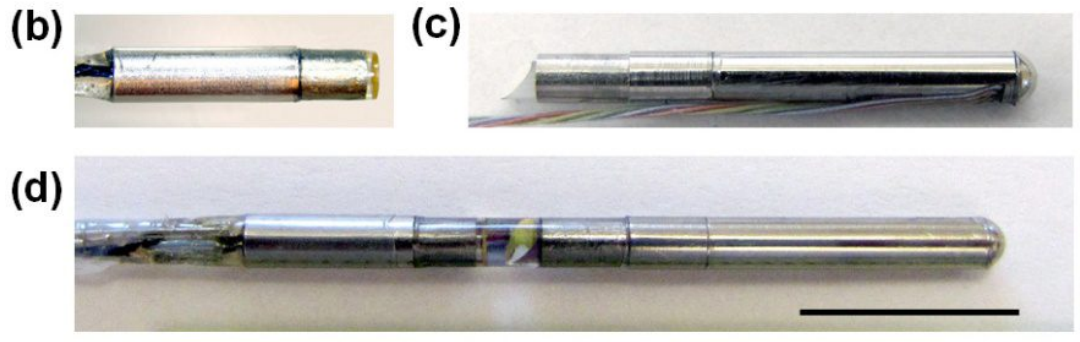

(e)

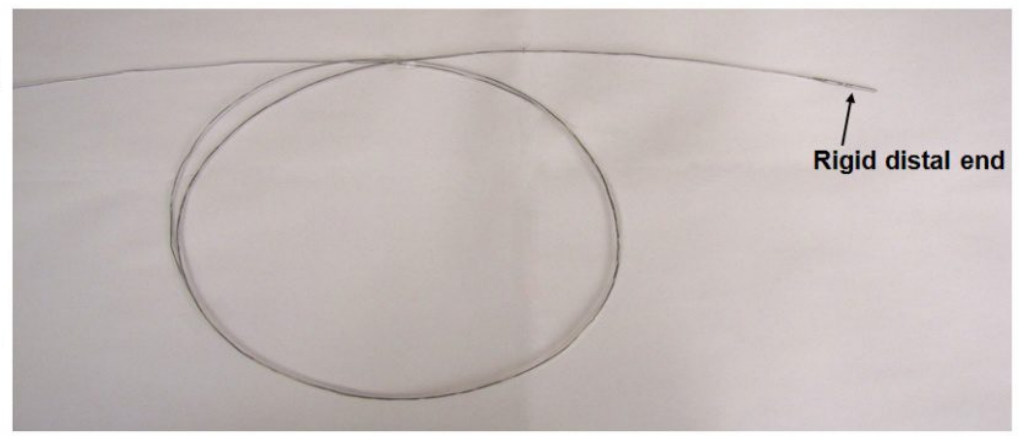

Figure 1. (a) Schematic of the PA endoscopic mini-probe. For acoustic matching, the endoscope's inner space is filled with a liquid medium (de-ionized water). However, the interior of the motor's housing compartment is isolated from the liquid medium to provide an in-air working condition, and the torque required for the scanning mirror rotation is transferred through magnetic coupling. (b) Optical fiber and US transducer unit. (c) Mechanical unit, including the scanning mirror and micromotor. (d) A photo showing the mini-probe's rigid distal end $(2.5 \mathrm{~mm}$ diameter and $\sim 35 \mathrm{~mm}$ length). The scale bar represents $1 \mathrm{~cm}$. (e) A photo showing the whole catheter, with a cord length of $\sim 2 \mathrm{~m}$. 
In the probe, we employed a focused US transducer fabricated using a highly-sensitive lead magnesium niobate-lead titanate (PMN-PT) for the piezoelectric-material to achieve adequate signal sensitivity and image resolution with the restricted aperture. We targeted a center frequency of around $40 \mathrm{MHz}$ and a focal distance of $\sim 4 \mathrm{~mm}$ for this transducer. The acoustic focusing was achieved by attaching a plano-concave plastic acoustic lens to the flat surface of the transducer's piezoelectric-crystal that has an outer diameter of $\sim 1.8 \mathrm{~mm}$ and inner diameter of $\sim 0.5 \mathrm{~mm}$. The plastic acoustic lens was fabricated in-house by molding a polyester resin. Building on the method described in ref. 4, we formed the spherical front surface with a radius of curvature of $\sim 1.5 \mathrm{~mm}$, which yielded an approximate focal length of $4 \mathrm{~mm}$. We chose the edge and center thickness of transducer to be as thin as possible, $\sim 0.4 \mathrm{~mm}$ and $\sim 0.1 \mathrm{~mm}$, respectively, to minimize the acoustic attenuation of high frequency acoustic waves.

For the animal imaging experiment reported here, we employed a $584 \mathrm{~nm}$ wavelength laser beam ( $10 \mathrm{~ns}$ pulse width) generated from a tunable dye laser (Cobra HRR, Sirah) pumped by a solid-state, diode-pumped Nd:YLF laser (INNOSLAB IS811-E, EdgeWave). We set the laser power at $\sim 0.04 \mathrm{~mJ} /$ pulse, which yielded an optical fluence of $\sim 2.3$ $\mathrm{mJ} / \mathrm{cm}^{2}\left(\sim 12 \%\right.$ of the ANSI safety limit ${ }^{25}$ for allowable skin laser fluence) near the plastic membrane. PA signals detected by the US transducer were amplified by an US pulser-receiver (5072 PR, Panametrics) and digitally recorded by a DAQ card (NI PCI-5124, National Instruments).

\subsection{Sensitivity measurement}

To quantify the sensitivity of the US transducer, we measured the insertion loss using the setup shown in Figure 2(a). To achieve this measurement, we sent electric pulses to the US transducer, which then generated acoustic pulses, and measured the electrically-converted acoustic echo signals that reflected from the flat surface of a quartz block located at the focal distance $(f)$ of the transducer. A transducer's insertion loss is defined as the logarithmic value of the voltage ratio of the received echo signal $\left(V_{p p}^{\text {Echo }}\right)$ to the input electric pulse $\left(V_{p p}^{\text {Input }}\right)$, i.e., $20 \log \left(V_{p p}^{\text {Echo }} / V_{p p}^{\text {Input }}\right)$. The input electric pulses for generating the acoustic pulse waves were sent in a burst mode, and we detected the time-domain voltage waveform of the echo signals by changing the central oscillation frequency $\left(f_{c}\right)$ of the input pulse and the object (i.e., quartz block) distance. To create the input electric pulses in the burst mode [Fig. 2(a)], we utilized a functional generator (33250A, Aligent) and isolation switch (ZASWA-2-50DR+, Mini-Circuits). By combining the continuous sinusoidal waveform provided by the function generator and square waves with a short duration (from another function generator), we could create the burst-mode input waveform. The main advantage of using such isolation switch is that it isolates the US transducer from the function generator during the receiving period (i.e., detecting the echo signals). An electrical schematic of the isolation switch is shown in Figure 2(b).

(a)

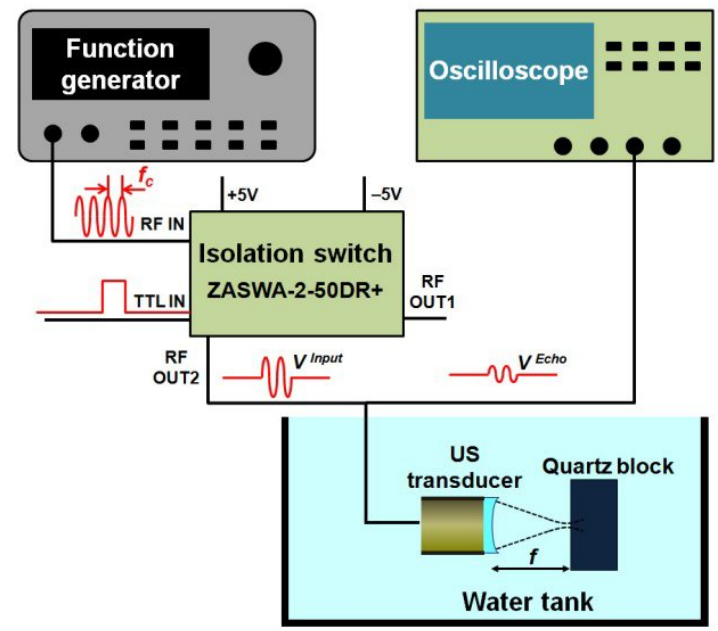

(b)

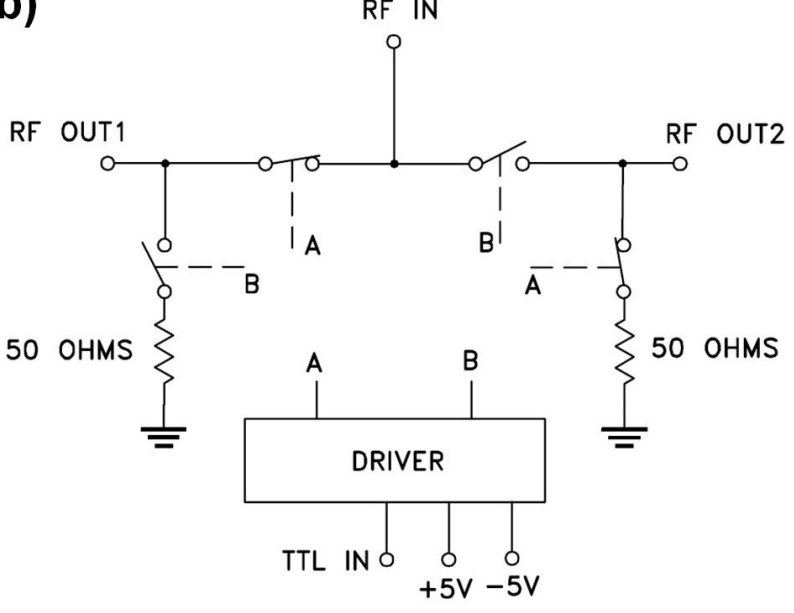

Figure 2. (a) A block diagram showing the experimental setup. (b) Electrical schematic of the isolation switch. 


\subsection{Imaging test with animal experiment}

To test the mini-probe's endoscopic imaging ability, we imaged the descending colon of an adult Sprague Dawley rat ( $\sim 50 \mathrm{~g}$; Harlan National Customer Service Center) ex vivo. To increase the likelihood of an empty colon for imaging, the rat was fasted for $\sim 12 \mathrm{hr}$ before the experiments. Before starting the imaging experiments, we first euthanized the animal by a pentobarbital overdose $(150 \mathrm{mg} / \mathrm{kg}$, IP). Once the animal was properly positioned, medical ultrasound gel was inserted into the colon via a small plastic tube. The ultrasound gel provided acoustic coupling between the tissue and US transducer and lubricated the probe during colon insertion through the anus. Then we inserted the endoscopic probe into the colon $\sim 4.5 \mathrm{~cm}$ deep from the animal's anus and performed pullback volumetric scans over a $\sim 4 \mathrm{~cm}$ range during constant pullback translation of the probe at a mechanically governed speed of $\sim 160 \mu \mathrm{m} / \mathrm{s}$. Each volumetric image required a scanning time of $\sim 4.2 \mathrm{~min}$. About $1000 \mathrm{~B}$-scan slices with longitudinal spacings of $\sim 40 \mu \mathrm{m}$ were acquired for each imaging mode.

All procedures in the experiment followed the protocol approved by the Institutional Animal Care and Use Committee at Washington University in St. Louis.

\section{RESULTS AND DISCUSSION}

\subsection{The insertion loss measurement result}

The US transducer showed a maximal echo response at an object distance of $\sim 4.1 \mathrm{~mm}$ and at a center frequency of $\sim 33$ $\mathrm{MHz}$ for the burst-mode input signal, which indicates the focal distance and center frequency of the transducer, respectively. The measured voltage amplitude of the echo signal was about $90 \mathrm{mV}$ (without any amplification) for an input electric pulse of $12 \mathrm{~V}$. This result yields a voltage ratio of $\sim 1 / 130$ and corresponding insertion loss of $-42 \mathrm{~dB}$. For the final insertion loss calculation, we compensated the signal loss from the attenuation in water $\left(2.2 \times 10^{-4}\right.$ $\left.\mathrm{dB} / \mathrm{mm} \cdot \mathrm{MHz}^{2}\right)$ and transmission into the quartz target $(-1.9 \mathrm{~dB})$ and acquired the final value of $-38 \mathrm{~dB}$. In Table 1, we present the PMN-PT transducer's experimentally-measured insertion loss result along with other relevant parameters. With the given parameters, we also simulated the US transducer's insertion loss by using modeling software (Field II) and calculated a theoretical value of $-11 \mathrm{~dB}$. This value could be regarded as a theoretical limit for such PMN-PT transducers fabricated with the given parameters. There was a significant difference between the experimental and theoretical results. We speculate that the high value of the experimental insertion loss might be primarily caused by the imperfect formation process of the plastic acoustic lens to the flat transducer.

Table 1. Specifications of the PMN-PT US transducer and its insertion loss.

\begin{tabular}{|c|c|c|c|c|c|c|c|c|}
\hline $\begin{array}{c}\text { Piezo- } \\
\text { material }\end{array}$ & Aperture & $\begin{array}{c}\text { Hole } \\
\text { dia. }\end{array}$ & $\begin{array}{c}\text { Focal } \\
\text { distance }\end{array}$ & $\begin{array}{c}\text { Acoustic } \\
\text { NA }\end{array}$ & $\begin{array}{c}\text { Center } \\
\text { freq. }\end{array}$ & Bandwidth & $\begin{array}{c}\text { Experimental } \\
\text { insertion loss }\end{array}$ & $\begin{array}{c}\text { Theoretical } \\
\text { insertion loss }\end{array}$ \\
\hline PMN-PT & $\sim 1.8 \mathrm{~mm}$ & $0.5 \mathrm{~mm}$ & $\sim 4.1 \mathrm{~mm}$ & $\sim 0.22$ & $\sim 33 \mathrm{MHz}$ & $\sim 20 \mathrm{MHz}(60 \%)$ & $-38 \mathrm{~dB}$ & $-11 \mathrm{~dB}$ \\
\hline
\end{tabular}

\subsection{Animal imaging result}

In Figure 3(a), we present a three-dimensionally rendered PA structural image acquired from the rat colon ex vivo. The image was processed from a volumetric data set covering a cylindrical volume $4 \mathrm{~cm}$ long with a $9 \mathrm{~mm}$ diameter. Since the data were acquired with a $584 \mathrm{~nm}$ laser wavelength, the PA signal amplitudes represent the total hemoglobin ( $\mathrm{Hb}$ and $\mathrm{HbO}_{2}$ ) concentration. The volume-rendered PA image shows the vasculature at the colon wall, which is around 1 mm thick. A radial-maximum amplitude projection (RMAP) image of Figure 3(a) is shown in Figure 3(b). The RMAP image shows the network of blood vessels in a two dimensional plane. Representative B-scan slices near the location indicated by the arrow in Figure 3(a) are presented in Cartesian [Fig. 3(c)] and polar [Fig. 3(d)] coordinates systems, respectively. 
(a)

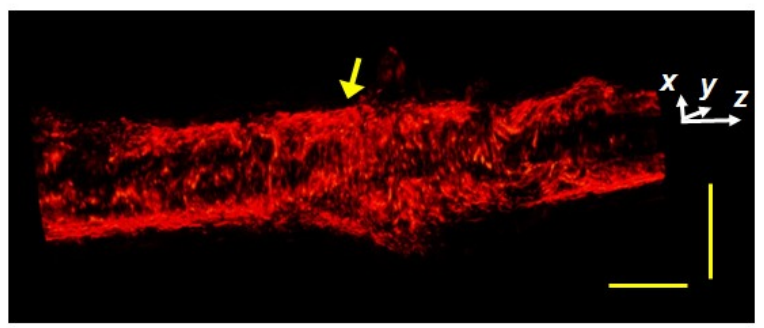

(b)

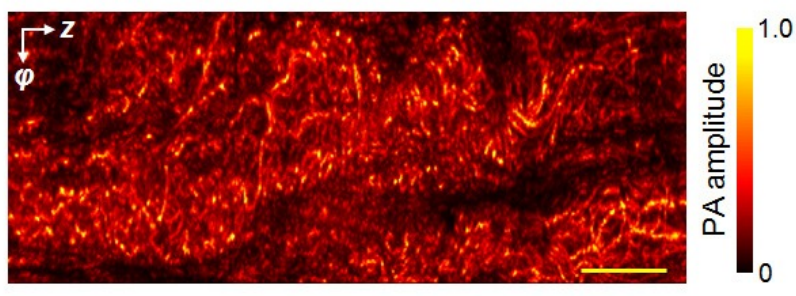

(c)

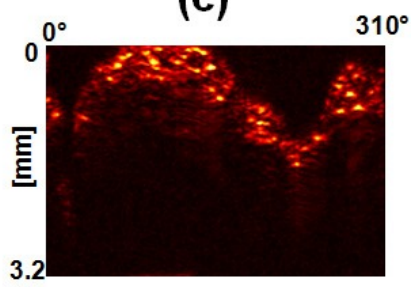

(d)

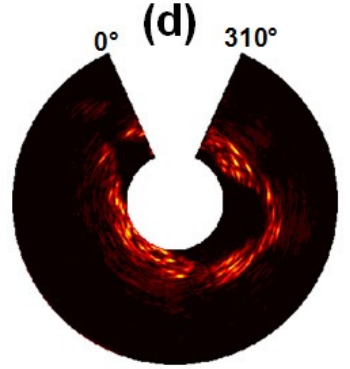

Figure 3. (a) Three-dimensionally-rendered PA structural image from a rat colon acquired ex vivo over a $4 \mathrm{~cm}$ range, with a $9 \mathrm{~mm}$ image diameter. The right-hand side $(+z)$ and lower portion $(-y)$ of the image correspond to the anus and the ventral side of the animal, respectively. The horizontal and vertical scale bars represent $5 \mathrm{~mm}$. (b) A PA-RMAP image of (a). The upper and lower parts of this image correspond to the left and right sides of the animal, and the middle section of the images are from the ventral side of the animal. The vertical $\varphi$-axis corresponds to the angular field-of-view covering $270^{\circ}$, and the horizontal $z$-axis corresponds to a pullback length of $4 \mathrm{~cm}$. Scale bars represent $5 \mathrm{~mm}$ (only for the horizontal direction). (c,d) A representative $x-y$ slice near the location indicated by the arrow in (a).

Although the new PMN-PT transducer's insertion loss was higher than typical values ${ }^{26,27}$, it showed an adequate PA signal sensitivity for colon vasculature imaging with a laser energy of $\sim 0.04 \mathrm{~mJ} / \mathrm{pulse}$, which yielded an optical fluence of $\sim 2.3 \mathrm{~mJ} / \mathrm{cm}^{2}(\sim 12 \%$ of the ANSI safety limit for allowable skin laser fluence).

\section{CONCLUSIONS}

We successfully implemented a 2.5-mm outer diameter PA endoscopic mini-probe using a PMN-PT US transducer. The 2.5-mm diameter includes the entire outer sheath, and it is smallest among the reported PA endoscopic probes that have been fully-catheterized. Also, we successfully tested the new mini-probe's endoscopic imaging capability through an ex vivo animal experiment, and could verify that the new PMN-PT transducer shows an adequate PA signal sensitivity for colon vasculature imaging.

\section{ACKNOWLEDGEMENT}

We thank Prof. James Ballard for his attentive reading of the manuscript. This work was sponsored in part by National Institutes of Health grants R01 CA157277, R01 NS46214 (BRP), R01 EB000712, R01 EB008085, and U54 CA136398 (Network for Translational Research). L.W. has a financial interest in Microphotoacoustics, Inc. and Endra, Inc., which, however, did not support this work. J.-M.Y. was supported in part by a Korea Research Foundation Grant funded by the Korean Government (KRF-2007-357-C00039).

\section{REFERENCES}

1. Wang, L.V. Prospects of photoacoustic tomography. Med Phys 35, 5758-5767 (2008).

2. Yang, J.M., et al. Photoacoustic endoscopy. Opt Lett 34, 1591-1593 (2009). 
3. Yang, J.M., et al. Volumetric photoacoustic endoscopy of internal organs: a phantom and in situ study. Proc. SPIE 7564, 75640D (2010).

4. Yang, J.M., et al. Volumetric photoacoustic endoscopy of upper gastrointestinal tract: ultrasonic transducer technology development Proc. SPIE 7899, 78990D (2011).

5. Oraevsky, A.A. \& Karabutov, A.A. Optoacoustic Tomography. in Biomedical Photonics Handbook, Vol. PM125 (ed. Vo-Dinh, T.) 3401-3434 (CRC Press, New York, 2003).

6. Wang, L.V. Multiscale photoacoustic microscopy and computed tomography. Nat Photonics 3, 503-509 (2009).

7. Wang, L.V. (ed.) Photoacoustic Imaging and Spectroscopy, (CRC, Boca Raton, 2009).

8. Li, C. \& Wang, L.V. Photoacoustic tomography and sensing in biomedicine. Phys Med Biol 54, R59-97 (2009).

9. Hu, S. \& Wang, L.V. Photoacoustic imaging and characterization of the microvasculature. J Biomed Opt 15, 011101 (2010).

10. Beard, P. Biomedical photoacoustic imaging. Interface Focus 1, 602-631 (2011).

11. Favazza, C.P., Cornelius, L.A. \& Wang, L.V. In vivo functional photoacoustic microscopy of cutaneous microvasculature in human skin. J Biomed Opt 16, 026004 (2011).

12. Chatni, M.R., et al. Functional photoacoustic microscopy of pH. J Biomed Opt 16, 100503 (2011).

13. Yao, J., Maslov, K.I., Shi, Y., Taber, L.A. \& Wang, L.V. In vivo photoacoustic imaging of transverse blood flow by using Doppler broadening of bandwidth. Opt Lett 35, 1419-1421 (2010).

14. Yao, J., Maslov, K.I., Zhang, Y., Xia, Y. \& Wang, L.V. Label-free oxygen-metabolic photoacoustic microscopy in vivo. J Biomed Opt 16, 076003 (2011).

15. Danielli, A., Favazza, C.P., Maslov, K. \& Wang, L.V. Single-wavelength functional photoacoustic microscopy in biological tissue. Opt Lett 36, 769-771 (2011).

16. Manohar, S., et al. Initial results of in vivo non-invasive cancer imaging in the human breast using near-infrared photoacoustics. Opt Express 15, 12277-12285 (2007).

17. Ermilov, S.A., et al. Laser optoacoustic imaging system for detection of breast cancer. J Biomed Opt 14, 024007 (2009).

18. Kruger, R.A., Lam, R.B., Reinecke, D.R., Del Rio, S.P. \& Doyle, R.P. Photoacoustic angiography of the breast. Med Phys 37, 6096-6100 (2010).

19. Steeg, P.S. Tumor metastasis: mechanistic insights and clinical challenges. Nat Med 12, 895-904 (2006).

20. Song, K.H., Stein, E.W., Margenthaler, J.A. \& Wang, L.V. Noninvasive photoacoustic identification of sentinel lymph nodes containing methylene blue in vivo in a rat model. $J$ Biomed Opt 13, 054033 (2008).

21. Erpelding, T.N., et al. Sentinel lymph nodes in the rat: noninvasive photoacoustic and US imaging with a clinical US system. Radiology 256, 102-110 (2010).

22. Dietrich, C. (ed.) Endoscopic Ultrasound: An Introductory Manual and Atlas, (Thieme, New York, 2006).

23. Menzel, J. \& Domschke, W. Gastrointestinal miniprobe sonography: the current status. Am J Gastroenterol 95, 605-616 (2000).

24. Wang, B., et al. Intravascular photoacoustic imaging. IEEE Journal of Selected Topics in Quantum Electronics 16, 588 - 599 (2010).

25. Laser Institute of America, American National Standard for Safe Use of Lasers ANSI Z136.1-2007 (American National Standards Institute, Inc., New York, NY, 2007). (2007).

26. Zhou, Q., et al. PMN-PT single crystal, high-frequency ultrasonic needle transducers for pulsed-wave Doppler application. IEEE Trans Ultrason Ferroelectr Freq Control 54, 668-675 (2007).

27. Peng, J., et al. PMN-PT single crystal thick films on silicon substrate for high-frequency micromachined ultrasonic transducers. Appl Phys A Mater Sci Process 2008, 161-163 (2008). 